\title{
STRONG CONSISTENCIES OF THE BOOTSTRAP MOMENTS
}

\author{
TIEN-CHUNG HU \\ Department of Mathematics \\ National Tsing Hua University \\ Hsinchu, Taiwan 30043, R.O.C. \\ and \\ Department of Statistics \\ University of North Carolina \\ Chapel Hill, NC 27599 U.S.A.
}

(Received November 14, 1990 and in revised form January 23, 1991)

ABSTRACT. Let $X$ be a real valued random variable with $E|X|^{r+\delta}<\infty$ for some positive integer $r$ and real number. $\delta, 0<\delta \leq r$, and let $\left\{x, x_{1}, x_{2}, \ldots\right\}$ be a sequence of independent, identically distributed random variables. In this note, we prove that. for almost all $w \in \Omega, \mu_{r ; n}^{*}(w) \rightarrow \mu_{r}$ with probability 1 , if $\lim _{n \rightarrow \infty} \inf m(n) n^{-\beta}>0$ for some $\beta>\frac{r-\delta}{r+\delta}$. where $\mu_{r ; n}^{*}$ is the bootstrap $r^{\text {th }}$ sample moment of the bootstrap sample with sample size $m(n)$ from the data set $\left\{x_{1}, x_{2} \ldots, x_{n}\right\}$ and $\mu_{r}$ is the $r^{\text {th }}$ moment of $X$. The results obtained here not only improve on those of Athreya [3] but also the proof is more elementary.

KEY WORDS AND PHRASES. Bootstrap sample size. Sample moment., Convergence with probability 1.

1980 AMS SUBJECT CLASSIFICATION OODES. Primary 60F15; secondary 62G05.

\section{INTRODUCTION}

Let $X$ be a real valued random variable with $E|X|^{r+\delta}<\infty$ for some positive real number $\delta \leq r$, and let $\left\{x, x_{1}, x_{2}, \ldots\right\}$ be a sequence of independent. Identically distributed random variables. Let

$$
F_{n}(x ; w)=\frac{1}{n} \sum_{i=1}^{n} I\left[X_{i}(w) \leq x\right], n=1,2 \ldots, w \in \Omega .
$$


be the empirical distribution functions associated with the sequence $\left\{x_{1}(w), x_{2}(w)\right.$. $\left.x_{3}(w) \ldots\right\}$. For every positive integer $n$ and $w \in \Omega$, let $\left\{x_{n 1}(w), x_{n 2}(w) \ldots \ldots\right.$ $\left.x_{n m(n)}(w)\right\}$ be independent, identically distributed random variables with distribution function $F_{n}(x: w)$ defined as in (1.1). We call $\left\{x_{n 1}(w), x_{n 2}(w), \ldots, x_{n m(n)}(w)\right\}$ the bootstrap sample set with bootstrap sample size $m(n)$; it is required that $m(n) \rightarrow \infty$ as $n \rightarrow \infty$. Denote by $\mu_{n ; r}(w)$ and $\mu_{n ; r}^{*}(w)$ the $r^{\text {th }}$ sample moment of $\left\{x_{1}(w)\right.$. $\left.x_{2}(w) \ldots, x_{n}(w)\right\}$ and the bootstrap $r^{\text {th }}$ sample moment of $\left\{x_{n 1}(x), x_{n 2}(w) \ldots\right.$ $\left.X_{n m(n)}(w)\right\}$ respectively and denote by $\mu_{r}$ the $r^{\text {th }}$ moment of $X$. (When $r=1$, we use $\mu_{n}(w)$ and $\mu_{n}^{*}(w)$ instead of $\mu_{n ; 1}(w)$ and $\mu_{n ; 1}^{*}(w)$ : further $\mu_{n}(w)$ and $\mu_{n}^{*}(w)$ are called sample mean and bootstrap sample mean respectively.) A problem. from the bootstrap theory of Efron [1]. is to find conditions such that, for almost all w, the bootstrap sample mean converges to the population mean (when it exists). That is, for almost all w.

$$
\mu_{\mathrm{n} ; \mathrm{r}}^{*}(w) \rightarrow \mu_{\mathrm{r}} \text { as } \mathrm{n} \rightarrow \infty
$$

with probability 1. By using the abstract "Vasserstein's metric" among distributions and a Mallow type inequality. Bickel and Freedman [2] showed that if $E|X|<\infty$, then for almost all $w \in \Omega$. (2) holds in probability. Athreya [3] found that if $E|X|^{\theta}<\infty$ for some $\theta \geq 1$, and $\lim \inf _{n \rightarrow \infty} m(n) n^{-\beta}>0$ for some $\beta>0$ such that $\theta \beta>1$. then for almost all $w \in \Omega$. (1.2) holds with probability 1 . To show this he used the difficult and complex inequality of Kurtz [4]. Bickel and Freedman and Athreya used deep mathematics and hard inequalities to prove the consistency of the bootstrap sample mean to the population mean. Their proofs are not easily comprehended. This note. provides an elementary way to obtain the strong consistency, relying on the Markov inequality. Moreover, the consistency property holds under weaker conditions than those presented in Athreya [3].

\section{RESULTS AND PROOFS}

THEOREM 2.1. Let $\left\{\mathrm{X}, \mathrm{X}_{1}, \mathrm{X}_{2}, \ldots\right\}$ be a sequence of independent, identically distributed random variables with $E|X|^{r+\delta}<\infty$ for some integer $r$ and real number $\delta S r$. Then, for almost all $w \in \Omega$. (1.2) holds with probability 1. If lim inf $m(n) n^{-\beta}>0$ for some real number $\beta>0$ such that $\beta>\frac{r-\delta}{r+\delta}$. $n \rightarrow \infty$

First, a lemma is needed in proving the theorem. The lemma is known in the literature. For the sake of completeness, a proof for the lemma is given.

LEMMA 2.2. Let $\left\{X, X_{1}, X_{2}, \ldots\right\}$ be a sequence of independent. Identically distributed random variables. Then, for any $0<p<1, E|X|^{p}<\infty$ implies that 


$$
\sum_{n=1}^{\infty} \frac{\left|x_{n}\right|}{n^{1 / p}}<\infty \quad \text { with probability } 1
$$

PROOF. Let $Y_{n}=\frac{\left|X_{n}\right|}{n^{1 / p}} I\left[\frac{\left|X_{n}\right|}{n^{1 / p}} \leq 1\right]$. Thus

$$
\begin{aligned}
\sum_{n=1}^{\infty} P\left[\frac{\left|X_{n}\right|}{n^{1 / p}} \neq Y_{n}\right] & =\sum_{n=1}^{\infty} P\left(\left|X_{n}\right|>n^{1 / p}\right) \\
& =\sum_{n=1}^{\infty} P\left(|X|^{P}>n\right)<\infty
\end{aligned}
$$

since $E|X|^{p}<\infty$. Defining $A_{j}=\left\{(j-1)^{1 / p}<|X| \leq(j)^{1 / p}\right\}$, we have, for $\alpha 21$.

$$
\begin{aligned}
\sum_{n=1}^{\infty} E\left(\left|Y_{n}\right|^{\alpha}\right) & =\sum_{n=1}^{\infty} \sum_{j=1}^{n} \int_{A_{j}} \frac{1}{n^{\alpha / p}}|x|^{\alpha} d P=\sum_{j=1}^{\infty}\left[\sum_{n=j}^{\infty} \frac{1}{n^{\alpha / p}}\right] \delta_{\Lambda_{j}}|x|^{\alpha} d P \\
S & \sum_{j=1}^{\infty}\left[\frac{C}{j(\alpha / p)-1}\right] \delta_{A_{j}}|x|^{\alpha} d P S \sum_{j=1}^{\infty} C \int_{A_{j}}|x|^{p}=C E|X|^{p}<\infty
\end{aligned}
$$

where the constant $\mathrm{C}$ depends only on $\alpha$ and p. Choosing $\alpha=1$ and 2, we have that

$$
\sum_{n=1}^{\infty} E\left|Y_{n}\right|<\infty
$$

and

$$
\sum_{n=1}^{\infty} \operatorname{Var} Y_{n}<\infty
$$

Thus, by the "three series Theorem" of Kolmogorov the lemma is established.

We are now in a position to prove the main result, which provides the strong consistency of the bootstrap sample moments.

PROOF OF THEOREM 2.1. It suffices to prove the result for the case $r=1$. The other cases can be proved in a similar way with minor changes. Recall that, for each $n$ and $w \in \Omega,\left\{x_{n 1}(w), x_{n 2}(w), \ldots, x_{n m(n)}(w)\right\}$ are the independent, identically distributed random variables with distribution function defined in (1.1). By the strong law of large numbers, we have for almost all $w \in \Omega$.

$$
\mu_{n}(w) \rightarrow \mu \text { as } n \rightarrow \infty \text {. }
$$

Thus, it suffices to show that, for almost all $w \in \Omega$.

$$
\left|\mu_{n}^{*}(w)-\mu_{n}(w)\right| \rightarrow 0 \text { as } n \rightarrow \infty
$$

wi th probability 1. From the Borel-Cantelli lemma, we only need to prove for almost all $w \in \Omega$ and for every $\epsilon>0$. 


$$
\sum_{n=1}^{\infty} P\left\{\left[\left|\frac{1}{m(n)} \sum_{i=1}^{m(n)} X_{n i}(w)-\mu_{n}(w)\right|>\epsilon\right] \mid x_{1}(w), x_{2}(w) \ldots . x_{n}(w)\right\}<\infty .
$$

For the case of presentation, we suppress all the symbol $w$ in $x_{1}(w) . X_{n i}(w)$ and $\mu_{n}(w)$ and the symbol $n$ in $m(n)$. Let $q$ be an integer such that $\frac{1}{q}<\beta-\frac{1-\delta}{1+\delta}$ and from the Markov inequality, we have

$$
P\left[\left|\frac{1}{m} \sum_{i=1}^{m} x_{n i}-\mu_{n}\right|>\epsilon \mid x_{1}, x_{2}, \ldots, x_{n}\right] \leq\left(\frac{1}{m \epsilon}\right)^{2 q} E\left[\left(\sum_{i=1}^{m}\left(x_{n i}-\mu_{n}\right)\right)^{2 q} \mid x_{1}, x_{2} \ldots . x_{n}\right]
$$

Now we write

$$
\begin{aligned}
& B_{2 q}=E\left[\left(\sum_{i=1}^{n}\left(x_{n i}-\mu_{n}\right)\right)^{2 q} \mid x_{1}, x_{2} \ldots x_{n}\right] \\
& =\sum_{i_{1}=1}^{m} \sum_{i_{2}=1}^{m} \cdots \sum_{i_{2 q}=1}^{m} E\left[\left(x_{n i_{1}}-\mu_{n}\right) \cdots\left(x_{n i_{2 q}}-\mu_{n}\right) \mid x_{1}, x_{2} \ldots x_{n}\right] \\
& =\sum_{\substack { t=1 \\
\begin{subarray}{c}{q_{1}+\ldots+q_{t}=2 q \\
q_{i} \geq 1, i=1 \ldots . t{ t = 1 \\
\begin{subarray} { c } { q _ { 1 } + \ldots + q _ { t } = 2 q \\
q _ { i } \geq 1 , i = 1 \ldots . t } }\end{subarray}}^{\sum} \frac{(2 q) !}{q_{1} ! \ldots q_{t} !}\left(\begin{array}{c}
m \\
t
\end{array}\right) E\left[\left(x_{n 1}-\mu_{n}\right)^{q_{1}} \ldots\left(x_{n t}-\mu_{n}\right)^{q_{t}} \mid x_{1}, x_{2} \ldots . x_{n}\right] \\
& =\sum_{t=1}^{q} \underset{q_{1}+\ldots+q_{t}=2 q}{\sum} \frac{(2 q) !}{q_{1} ! \ldots q_{t} !}\left({ }_{t}^{m}\right) E\left[\left(x_{n 1}-\mu_{n}\right)^{q_{1}} \mid x_{1}, x_{2} \ldots x_{n}\right] \\
& q_{i} 22,1=1, \ldots, t \\
& \ldots E\left[\left(x_{n t}-\mu_{n}\right)^{q_{t}} \mid x_{1}, x_{2}, \ldots, x_{n}\right]
\end{aligned}
$$

where the third equality in (2.4) holds since $x_{n 1}, x_{n 2} \ldots, x_{n m}$ are identically distributed. Further the last equality in (2.4) is justified since $x_{n 1}, x_{n 2} \ldots, x_{n m}$ are independent and $E\left(X_{n 1}-\mu_{n}\right)=0$ implies that there is no contribution for those terms which contain at least one of $q_{i}=1$. In the sequel, we use the shorthand notation $a_{n} \sim b_{n}$ for $a_{n}=O\left(b_{n}\right)$ as $n \rightarrow \infty$. Thus, from (2.4) we have

$$
\begin{aligned}
B_{2 q} & \sim \sum_{t=1}^{q} m^{t} E\left[\left(x_{n 1}-\mu_{n}\right)^{q_{1}} \mid x_{1}, x_{2} \ldots x_{n}\right] \cdots E\left[\left(x_{n t}-\mu_{n}\right)^{q} t \mid x_{1}, x_{2} \ldots \ldots x_{n}\right] \\
& =\sum_{t=1}^{q} m^{t}\left[\frac{1}{n} \sum_{i=1}^{n}\left(x_{i}-\mu_{n}\right)^{q_{1}}\right] \ldots\left[\frac{1}{n} \sum_{i=1}^{n}\left(x_{i}-\mu_{n}\right)^{q_{t}}\right] \\
& =\sum_{t=1}^{q}\left(\frac{m}{n}\right)^{t}\left[\sum_{i=1}^{n}\left(x_{i}-\mu_{n}\right)^{q_{1}}\right] \cdots\left[\sum_{i=1}^{n}\left(x_{i}-\mu_{n}\right)^{q_{t}}\right]
\end{aligned}
$$




$$
\begin{gathered}
s \sum_{t=1}^{q}\left(\frac{m}{n}\right)^{t}\left[\sum_{i=1}^{n} 2^{q_{1}}\left(\left|x_{i}\right|^{q_{1}}+\left|\mu_{n}\right|^{q_{1}}\right)\right] \cdots\left[\sum_{i=1}^{n} 2^{q_{t}}\left(\left|x_{i}\right|^{q_{t}}+\left|\mu_{n}\right|^{q_{t}}\right)\right] \\
\left.s 2^{2 q} \sum_{t=1}^{q}\left(\frac{m}{n}\right)^{t}(n) \sum_{j=1}^{t} \frac{q_{j}}{q_{j}-1+q} \sum_{i=1}^{n}\left(\frac{1}{i}\right)^{\frac{q_{1}}{q_{1}-1+\delta}}\left(\left|x_{i}\right|^{q_{1}}+\left|\mu_{n}\right|^{q_{1}}\right)\right] \\
\cdots\left[\sum_{i=1}^{n}\left(\frac{1}{i}\right)^{\frac{q_{t}}{q_{t}-1+\delta}}\left(\left|x_{i}\right|^{q_{t}}+\left|\mu_{n}\right|^{q_{t}}\right)\right]
\end{gathered}
$$

since $q_{j} \geq 2, q_{1}+q_{2}+\ldots+q_{t}=2 q$ and where the first inequality in (2.5) is obtained for fact that $|a+b|^{s} \leq 2^{s}\left(|a|^{s}+|b|^{s}\right)$ for $a, b$ and $s$ real numbers. Since $\mu$ is finite it follows from (2.1) that, for almost all $w$. there exists a constant $C$ such that $\mu_{n}<C$ for every $n$. Further note that $\delta<1$ and $q_{j} \geq 2$ for $j=1,2 \ldots, q$ which imply $\frac{q_{j}}{q_{j}-1+\delta}>1$. Thus, for almost all $w \in \Omega$.

$$
\sum_{i=1}^{\infty}\left(\frac{1}{i}\right)^{\frac{q_{j}}{q_{j}-1+\delta}}\left|\mu_{n}\right|^{q_{j}}<\infty . \quad j=1.2 \ldots . q .
$$

Now applying our lemma to $\left|x_{i}\right|^{q_{j}}$ and choosing $p=\frac{q_{j}-1+\delta}{q_{j}}<1$, we have, for almost all $w \in \Omega$.

$$
\sum_{i=1}^{\infty}\left(\frac{1}{i}\right)^{\frac{q_{j}}{q_{j}-1+\delta}}\left|x_{i}\right|^{q_{j}}<\infty . j=1.2 \ldots . q .
$$

Without loss of generality we put $m(n)=n^{\beta}$ where $\beta$ is some real number such that $\beta$ > $\frac{1-\delta}{1+\delta}$, then from (2.5). (2.6) and (2.7), we have

$$
\left(\frac{1}{m}\right)^{2 q} B_{2 q} \sim \sum_{t=1}^{q}\left[\frac{1}{n}\right]^{2 q \beta+(1-\beta) t \Sigma_{j=1}^{t}} \frac{q_{j}}{q_{j}^{-1+\delta}}
$$

Denote the exponent of $\left(\frac{1}{n}\right)$ in $(2.8)$ as $\phi(t)$ for $t=1,2 \ldots . q$. Note that

$$
\begin{aligned}
\phi(t) & =2 q \beta+(1-\beta) t-\sum_{j=1}^{t} \frac{q_{j}}{q_{j}-1+\delta} \\
& 22 q \beta-t\left(\beta-1+\frac{2}{1+\delta}\right)
\end{aligned}
$$




$$
\begin{aligned}
& 22 \mathrm{q} \beta-\mathrm{q}\left(\beta+\frac{1-\delta}{1+\delta}\right) \\
& =\mathrm{q}\left(\beta-\frac{1-\delta}{1+\delta}\right)
\end{aligned}
$$

for $t=1.2 \ldots, q$. where $(2.9)$ holds since $\frac{q_{j}}{q_{j}-1+\delta}<\frac{2}{1+\delta}$ for $j=1,2 \ldots, t$. By the appropriate choice of $q .(2.2)$ follows from (2.3), (2.8) and (2.10). This completes the proof.

Theorem 1 of Athreya, stated in Corollary 2.3, is an immediate consequence of our Theorem 2.1.

OOROLLARY 2.3. If $E|X|<\infty$ and $m(n)=p^{n}$ for some $\rho>1$, then (2.2) holds, for $r=1$.

COROLLARY 2.4. If $E X^{2}<\infty$ and $m(n)=n^{\beta}$ for some $\beta>0$, then (2.2) holds for $\mathbf{r}=1$.

PROOF. By letting $\delta=1-\alpha$ for some $\alpha<\frac{2 \beta}{1+\beta}$.

REMARKS. Let $m(n)$ growth with an algebraic rate: that is, $m(n)=n^{\beta}$ for some $\beta>0$. First note that $1+\delta$ in our notation plays the role of $\theta$ in Athreya's. In case $1<\theta<2$ we have $\beta>\frac{1}{1+\delta}>\frac{1-\delta}{1+\delta}$. Hence, in this case, our condition is strictly weaker than the one is posed by Athreya. In case $\theta 22$. we only require $\beta>0$ which is even much weaker than $\beta>\frac{1}{\theta}$ the requirement in the Theorem 2 of Athreya. However. if $\theta=1$, then both of our theorem and Athreya's theorem require $\beta>1$.

Recently. the author learned that Professor Csorgo also improved the result of Athreya using a different approaching.

ACKNOWLECEMENT. The author is most grateful to the Department of Statistics. University of North Carolina. Chapel Hill, for their hospitality. The author also wishes to thank the editor and a referee for helpful suggestions.

\section{REFERENCES}

1. Efron. B. Bootstrap methods: Another look at the jacknife. Annals of Statistics 7 (1979), 1-26.

2. Bickel. P.J. and Freedman, D.A. Some asymptotic theory for the bootstrap. Annals of Stattstics 9 (1981). 1196-1217.

3. Athreya. K.B. Strong law for bootstrap. Statistics and Probability Letters 1 (1983). 147-150.

4. Kurtz. T.G. Inequalities for laws of large numbers. Annals of Mathematical Statistics 43 (1972), 1874-1883.

5. Chung. K.L. $\Lambda$ Course in Probability Theory. Harcourt. Brace and World. New York. 1968. 


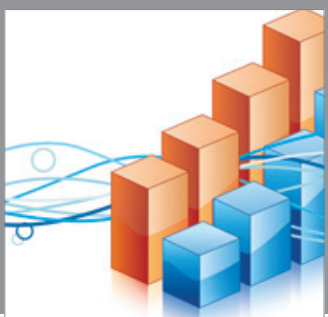

Advances in

Operations Research

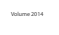

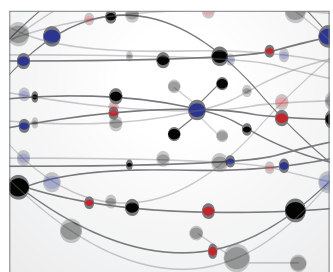

\section{The Scientific} World Journal
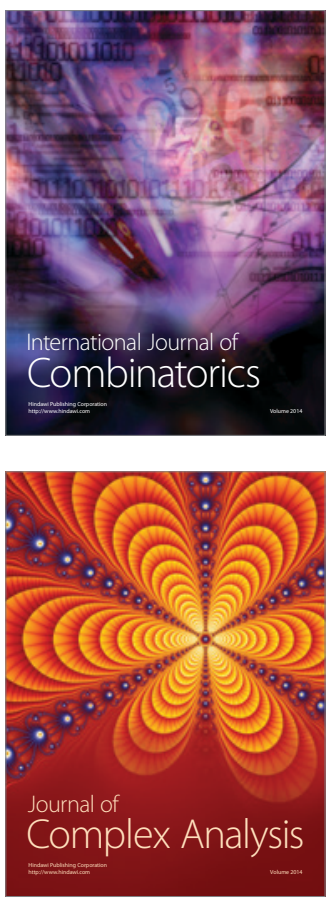

International Journal of

Mathematics and

Mathematical

Sciences
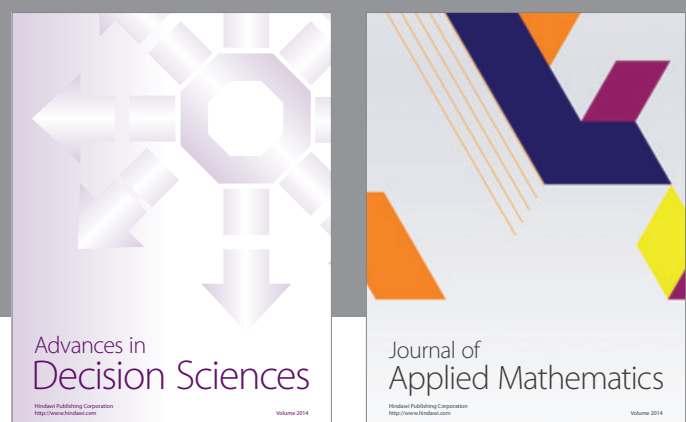

Journal of

Applied Mathematics
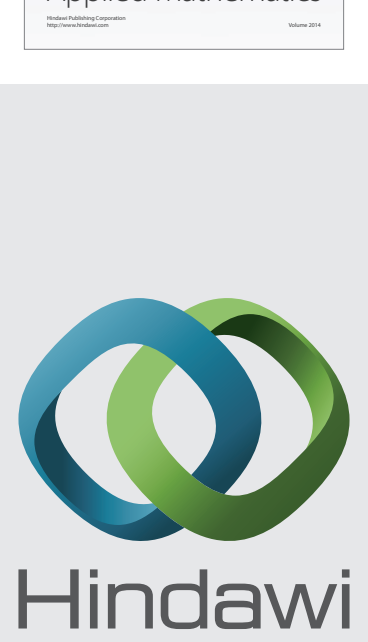

Submit your manuscripts at http://www.hindawi.com
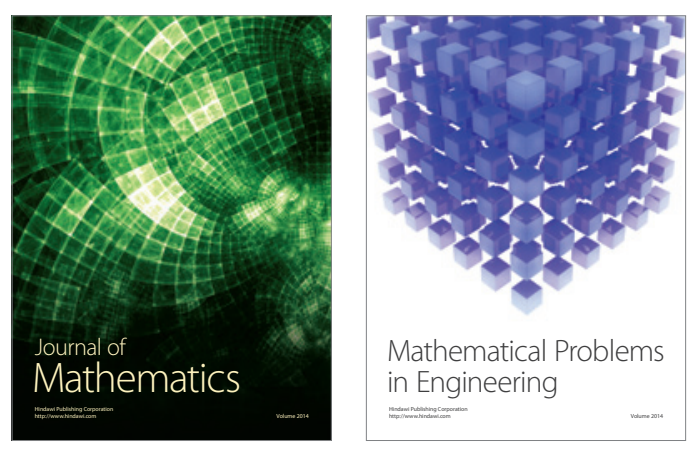

Mathematical Problems in Engineering
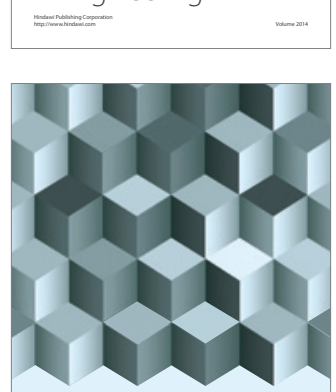

Journal of

Function Spaces
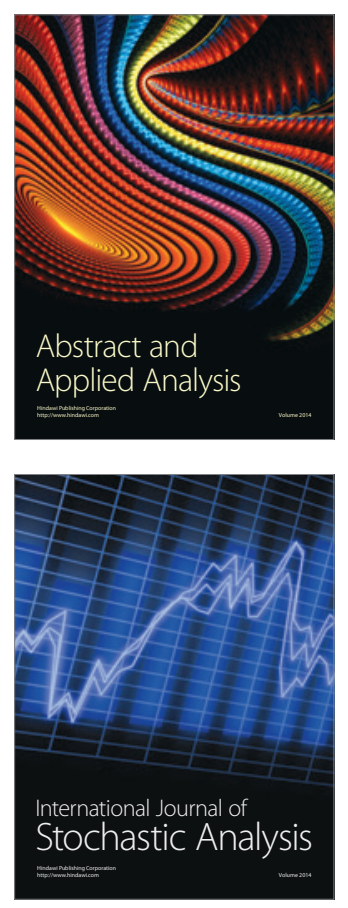

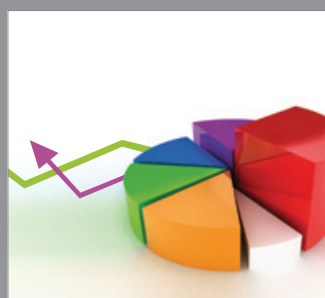

ournal of

Probability and Statistics

Promensencen
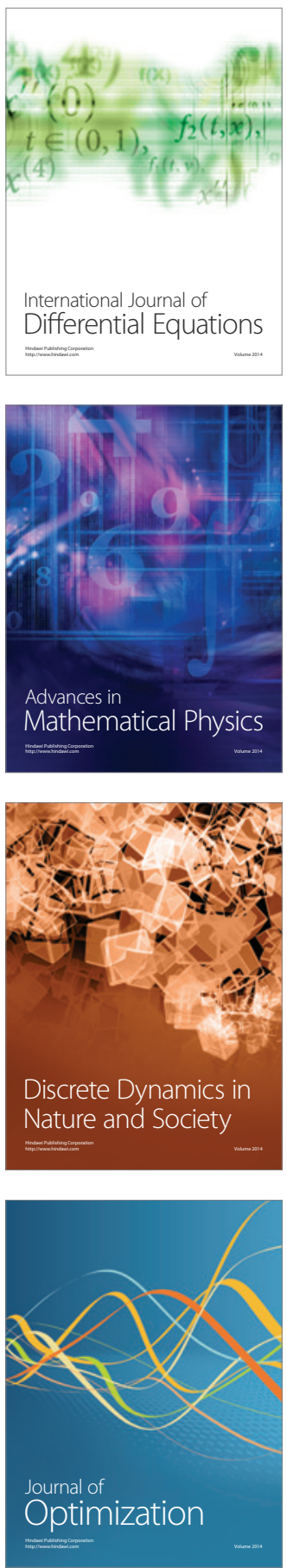